\title{
Alteration of p53-binding protein 1 expression as a risk factor for local recurrence in patients undergoing resection for extrahepatic cholangiocarcinoma
}

\author{
TOSHIFUMI WAKAI ${ }^{1}$, YOSHIO SHIRAI $^{1}$, JUN SAKATA $^{1}$, PAVEL V. KORITA $^{2}$, \\ YASUNOBU MATSUDA ${ }^{3}$, MASAAKI TAKAMURA ${ }^{3}$, RIUKO OHASHI ${ }^{4}$, \\ MASAYUKI NAGAHASHI ${ }^{1}$, YOICHI AJIOKA ${ }^{2}$ and KATSUYOSHI HATAKEYAMA ${ }^{1}$
}

\begin{abstract}
Divisions of ${ }^{1}$ Digestive and General Surgery, ${ }^{2}$ Molecular and Diagnostic Pathology, ${ }^{3}$ Gastroenterology
and Hepatology, ${ }^{4}$ Cellular and Molecular Pathology, Niigata University Graduate School of Medical

and Dental Sciences, 1-757 Asahimachi-dori, Chuo-ku, Niigata 951-8510, Japan
\end{abstract}

Received December 17, 2010; Accepted February 7, 2011

DOI: 10.3892/ijo.2011.959

\begin{abstract}
P53-binding protein 1 (53BP1) is an early DNA damage response-protein that is rapidly recruited to sites of DNA double-strand breaks. The presence of 53BP1 nuclear foci can be considered as a cytologic marker for endogenous double-strand breaks reflecting genomic instability. This study aimed to clarify the early DNA damage response mediated by 53BP1 in tumor specimens of ductal resection margins and to elucidate its predictive value for clinically evident local recurrence at ductal stumps in 110 patients undergoing resection for extrahepatic cholangiocarcinoma. The ductal resection margin status was classified as negative ( 85 patients), positive with carcinoma in situ (14 patients), or positive with invasive carcinoma (11 patients). The nuclear staining pattern of 53BP1 was evaluated by immunofluorescence. TUNEL analysis was used to calculate apoptotic index. Ductal margin status was the only independent risk factor for local recurrence $(\mathrm{P}=0.001)$. The cumulative probability of local recurrence at 5 years was $10 \%, 40 \%$ and $100 \%$ in patients with negative ductal margins, positive with carcinoma in situ and positive with invasive carcinoma, respectively $(\mathrm{P}<0.001)$. Of the 14 tumor specimens of carcinoma in situ, 10 showed diffuse localization of 53BP1 in nuclei (53BP1 inactivation) and 4 showed discrete nuclear foci of 53BP1 (53BP1 activation). All 11 tumor specimens of invasive carcinoma showed 53BP1 inactivation. Apoptotic index was markedly decreased in tumor specimens with 53BP1 inactivation compared to those with 53BP1 activation (median
\end{abstract}

Correspondence to: Dr Toshifumi Wakai, Division of Digestive and General Surgery, Niigata University Graduate School of Medical and Dental Sciences, 1-757 Asahimachi-dori, Chuo-ku, Niigata 951-8510, Japan

E-mail: wakait@med.niigata-u.ac.jp

Key words: cholangiocarcinoma, extrahepatic bile duct carcinoma, p53-binding protein 1 , immunofluorescence, prognosis index, $0 \%$ vs. $22 \%$; $\mathrm{P}<0.001)$. Among 14 patients with residual carcinoma in situ, the cumulative probability of local recurrence was significantly higher in patients with 53BP1 inactivation than in patients with 53BP1 activation $(60 \%$ vs. $0 \%$ at 5 years; $\mathrm{P}=0.020)$. In conclusion, after resection for extrahepatic cholangiocarcinoma, clinically evident local recurrence at ductal stumps is closely associated with 53BP1 inactivation and decreased apoptosis.

\section{Introduction}

A DNA double-strand break (DSB) is a serious lesion that can initiate genomic instability, ultimately leading to cancer (1). A key component in DNA damage response is the histone protein $\mathrm{H} 2 \mathrm{AX}$, which becomes rapidly phosphorylated on a serine, four residues from the carboxyl terminus, to form $\gamma \mathrm{H} 2 \mathrm{AX}$ at nascent DSB sites (1). Thus, histone $\mathrm{H} 2 \mathrm{AX}$ phosphorylation is a sensitive marker for DSBs (1-3). Recent evidence suggests that p53-binding protein 1 (53BP1) has a role in maintaining genomic stability during long-range joining of DNA DSBs (2). As 53BP1 is an early DNA damage response-protein that is rapidly recruited to sites of DSBs and binds the phosphorylated tail of $\gamma \mathrm{H} 2 \mathrm{AX}$ (1-4), the presence of 53BP1 nuclear foci can be considered as a cytologic marker for endogenous DSBs reflecting genomic instability $(5,6)$. Recent studies proposed that immunofluorescence analysis of 53BP1 expression can be a useful tool to estimate the level of genomic instability and malignant potential in precancerous lesions arising from the lung (3), thyroid (5) and skin (6).

After surgical resection for extrahepatic cholangiocarcinoma, invasive carcinoma at the ductal resection margins has a strong adverse effect on patient survival, whereas residual carcinoma in situ does not (7-13). Although a small proportion of patients with residual carcinoma in situ at ductal stumps survive in the long term, others develop late local recurrence (7-16). In contrast, some patients with residual carcinoma in situ at ductal stumps die of local recurrence within 5 years after resection (7-13). These findings suggest that a closer investigation of the molecular mechanisms involved in these residual tumors 
at ductal stumps is warranted. Therefore, we hypothesized that the early DNA damage response mediated by 53BP1 in tumor specimens of ductal resection margins may affect the long-term outcome of patients undergoing surgical resection for extrahepatic cholangiocarcinoma. This retrospective study sought to clarify the early DNA damage response mediated by 53BP1 in tumor specimens of ductal resection margins and to elucidate its predictive value for clinically evident local recurrence at ductal stumps.

\section{Materials and methods}

Patient population. From January 1988 to December 2007, a total of 114 consecutive Japanese patients with carcinoma arising from the extrahepatic bile ducts (extrahepatic cholangiocarcinoma) underwent surgical resection with curative intent at the Niigata University Medical and Dental Hospital. Four patients who also had gallbladder carcinoma were excluded. The remaining 110 patients $(84$ of whom were included in our earlier report) (7) formed the basis of the current retrospective study, which included 69 men and 41 women with a median age of 66 years (range 35-86 years). The study protocol was approved by the Institutional Review Board of Niigata University Medical and Dental Hospital, and written informed consent was obtained from all patients involved in the current study.

Surgical resection procedures. Surgical resection procedures depended on the location of the primary tumor. Fifty-seven patients underwent a hepatectomy with extrahepatic bile duct resection, 29 underwent a Whipple procedure or a pyloruspreserving pancreaticoduodenectomy, 12 underwent an extrahepatic bile duct resection, and a combined hepatectomy and pancreaticoduodenectomy was performed in 12 patients. All 110 patients also underwent dissection of the regional lymph nodes, which included the pericholedochal, retroportal, cystic duct, right celiac, posterosuperior pancreaticoduodenal, and hepatic artery node groups (17-20). In patients undergoing a pancreaticoduodenectomy, the right portion of the superior mesenteric nodes (20) was also dissected. Seventy-three patients with suspected regional nodal disease underwent dissection of the paraaortic lymph nodes in addition to regional lymphadenectomy, and 6 had involvement of the paraaortic lymph nodes, which was classified as pathologic distant metastasis (pM1) (17). In the current series, no patients underwent a portal vein embolization before resection.

Complications after resection occurred in 67 (61\%) patients. Three patients died within 30 days of resection, giving an operative mortality rate of $2.7 \%$. Adjuvant treatment after surgical resection was administered at the discretion of the individual surgeon. Thirty-three patients received adjuvant treatment: oral or intravenous administration of 5-fluorouracil (21 patients) and intravenous administration of gemcitabine alone ( 8 patients). The remaining 4 patients with positive ductal resection margins received adjuvant local radiotherapy with (2 patients) or without (2 patients) systemic chemotherapy.

Patient follow-up after resection. Patients undergoing a surgical resection were followed regularly in outpatient clinics every 3 to 6 months, with a median follow-up period of 99 months (range, 1 to 259 months). At the time of disease status assessment, 60 patients had died of tumor recurrence and 3 patients had died of suppurative cholangitis. Seventeen patients had died of other causes with no evidence of tumor recurrence. Six patients were alive with recurrent disease and the remaining 24 patients were alive with no evidence of disease. The site of initial disease recurrence was determined by contrast-enhanced computed tomography and/or cholangiography after percutaneous transhepatic biliary drainage. Local recurrence was defined as that occurring at the ductal stumps.

Pathologic evaluation. Resected specimens were submitted to the Department of Surgical Pathology in our hospital for histologic evaluation. Histologic findings were described according to the American Joint Committee on Cancer (AJCC) Staging Manual (17). The extent of the primary tumor was determined through the examination of multiple sections (median of 10 sections; range, 2 to 49 sections) of the entire lesion in each resected specimen. The location of the primary tumor was classified as hilar or nonhilar according to the classification of Bismuth et al $(21,22)$. The median tumor size was $40 \mathrm{~mm}$ (range, 10 to $100 \mathrm{~mm}$ ). Histopathologic type was classified as papillary or non-papillary $(17,23,24)$. Histologic grade was based on the area of the tumor having the highest grade (17). Adenocarcinoma was identified as the primary tumor in 107 patients and adenosquamous carcinoma was identified in 3 patients. A total of 2728 lymph nodes taken from the 110 patients who underwent a surgical resection (median of 23 lymph nodes per patient) were examined histologically for metastases. A representative $3-\mu \mathrm{m}$ section was cut from each lymph node and stained with hematoxylin and eosin.

In the current study, severe dysplasia was included in the category of carcinoma in situ, because it is usually difficult or even impossible to distinguish between these two epithelial lesions $(7,23,24)$. Ductal resection margin status was assessed histologically by frozen-section examination of ductal stumps in 95 of $110(86 \%)$ patients during the operation. After reviewing both frozen sections of ductal stumps and routinely-stained tissue sections of resected specimens, ductal resection margin status was determined. The continuity of carcinoma in situ at ductal resection margins from the main tumor was confirmed on multiple tissue sections. The ductal resection margin status was classified into negative, positive with carcinoma in situ, or positive with invasive carcinoma. A positive ductal margin with both carcinoma in situ and invasive carcinoma was classified as positive with invasive carcinoma.

Analysis of tissue samples. In cases with positive ductal margins, formalin-fixed paraffin-embedded blocks of main tumors and positive ductal margins with carcinoma in situ or invasive carcinoma were used for immunohistochemistry, immunofluorescence and terminal deoxynucleotidyl transferasemediated dUTP nick-end labeling (TUNEL) analysis. For normal controls, 7 samples of non-neoplastic biliary epithelium surrounding tumor from 7 patients without jaundice who underwent no preoperative biliary decompression procedure were evaluated. Nine serial $3-\mu \mathrm{m}$ tissue sections were re-cut and prepared from each block as follows: 1 for hematoxylin-eosin staining, 4 for immunohistochemical staining with the following antibodies: $\gamma \mathrm{H} 2 \mathrm{AX}$ antibody (Bethyl Laboratories Inc., 
Table I. Factors significantly associated with long-term survival after resection.

\begin{tabular}{|c|c|c|c|c|c|c|}
\hline \multirow[b]{2}{*}{ Variable } & \multirow{2}{*}{$\begin{array}{l}\text { No. of } \\
\text { patients }\end{array}$} & \multicolumn{2}{|c|}{$\begin{array}{l}\text { Survival } \\
\text { rate }(\%)\end{array}$} & \multirow{2}{*}{$\begin{array}{l}\text { Univariate } \\
\text { analysis } \\
\text { P-value }\end{array}$} & \multicolumn{2}{|c|}{$\begin{array}{c}\text { Multivariate } \\
\text { analysis }\end{array}$} \\
\hline & & 5-year & 10-year & & Relative risk $(95 \% \mathrm{CI})$ & P-value \\
\hline Size of the primary tumor (mm) & & & & 0.016 & & \\
\hline$<40$ & 51 & 56 & 40 & & & \\
\hline$\geq 40$ & 59 & 31 & 25 & & & \\
\hline pN classification & $<0.0$ & & & & & \\
\hline pNO & 65 & 57 & 43 & & 1.00 & \\
\hline $\mathrm{pN} 1$ & 45 & 20 & 13 & & $1.79(1.04-3.10)$ & 0.037 \\
\hline pM classification & & & & $<0.001$ & & \\
\hline pM0 & 104 & 44 & 31 & & 1.00 & \\
\hline $\mathrm{pM} 1$ & 6 & 0 & 0 & & $.01-16.83)$ & 0.001 \\
\hline \multicolumn{7}{|l|}{ Histologic grade } \\
\hline G1 & 27 & 59 & 50 & & & \\
\hline $\mathrm{G} 2$ & 40 & 40 & 26 & & & \\
\hline G3 & 43 & 31 & 23 & & & \\
\hline \multicolumn{7}{|l|}{ Blood vessel invasion } \\
\hline Absent & 57 & 56 & 44 & & & \\
\hline Present & 53 & 29 & 22 & & & \\
\hline Ductal margin status & & & & 0.003 & & 0.029 \\
\hline Negative & 85 & 47 & 39 & & 1.00 & \\
\hline Positive with carcinoma in situ & 14 & 46 & 15 & & $1.16(0.54-2.48)$ & 0.706 \\
\hline Positive with invasive carcinoma & & 0 & 0 & & $3.08(1.43-6.63)$ & 0.004 \\
\hline
\end{tabular}

$\mathrm{CI}$, confidence interval; $\mathrm{pN}$ classification, pathologic lymph node metastasis classification; pM, pathologic distant metastasis; G1, well differentiated; G2, moderately differentiated; G3, poorly differentiated.

Montgomery, TX; 1:200 dilution), 53BP1 antibody (Bethyl Laboratories Inc.; 1:200 dilution), p53 monoclonal antibody (Leica Microsystems, Newcastle-upon-Tyne, UK; 1:200 dilution), or Ki-67 monoclonal antibody (Dako, Glostrup, Denmark; 1:200 dilution), 1 for TUNEL analysis, 1 for immunofluorescence with 53BP1 antibody, 1 for double-labeled immunofluorescence with $\gamma \mathrm{H} 2 \mathrm{AX}$ and 53BP1 antibodies, and 1 as a negative control. Immunohistochemistry was performed using the streptavidin-biotin immunoperoxidase method with the Histofine SAB-PO kit (Nichirei Biosciences, Tokyo, Japan). Apoptotic cells were identified using an Apop Tag ${ }^{\circledR}$ Peroxidase In Situ Apoptosis Detection kit (Millipore Co., Billerica, MA). All of the non-neoplastic biliary epithelial cells in normal controls and tumor cells in which the reaction product was found in the nuclei were scored as positive, regardless of the staining intensity. Estimation of the nuclear labeling index was based on the percentage of labeled nuclei in the area of tumor cells at the ductal resection margins. The expression of p53 was classified into 4 patterns: negative, sporadic, focal, and diffuse. Focal and diffuse expression patterns were deemed to represent overexpression of p53 as described previously (25). Two independent pathologists blinded to the clinical details assessed each section.
To evaluate the DNA damage response mediated by 53BP1, the nuclear staining pattern of 53BP1 was examined by immunofluorescence. Paraffin sections were deparaffinized and heated in sodium citrate buffer at $\mathrm{pH} 6.5$ for $20 \mathrm{~min}$ at $121^{\circ} \mathrm{C}$. The specimens were blocked with $10 \%$ normal goat serum for $30 \mathrm{~min}$, then sections were incubated with 53BP1 antibody (1:50 dilution) for $24 \mathrm{~h}$ at room temperature, and were labeled with Alexa Fluor 488 F(ab')2 fragment of goat anti-rabbit IgG (Molecular Probes, Eugene, OR) for $1 \mathrm{~h}$ at a dilution of 1:500. For double-labeled immunofluorescence with $\gamma \mathrm{H} 2 \mathrm{AX}$ and 53BP1 antibodies, after blocking, sections were incubated with $\gamma \mathrm{H} 2 \mathrm{AX}$ antibody (1:50 dilution) for $24 \mathrm{~h}$ at room temperature, and then labeled with Alexa Fluor $568 \mathrm{~F}\left(\mathrm{ab}^{\prime}\right) 2$ fragment of goat anti-rabbit IgG (Molecular Probes) for $1 \mathrm{~h}$ at a dilution of 1:100. After labeling for $\gamma \mathrm{H} 2 \mathrm{AX}$, the sections were then incubated with 53BP1 antibody labeled with a Zenon ${ }^{\mathrm{TM}}$ Alexa Fluor $^{\circledR} 488$ Rabbit IgG Labeling kit (Molecular Probes) for $2 \mathrm{~h}$ at a final dilution of 1:25, in order to detect 53BP1. Primary antibodies were omitted in control experiments. After completing the staining procedures, sections were covered with a few drops of Vectashield Mounting Medium with DAPI (Vector Laboratories Inc., Burlingame, CA) and dried at room temperature before examination. Immunofluorescence was captured with a 


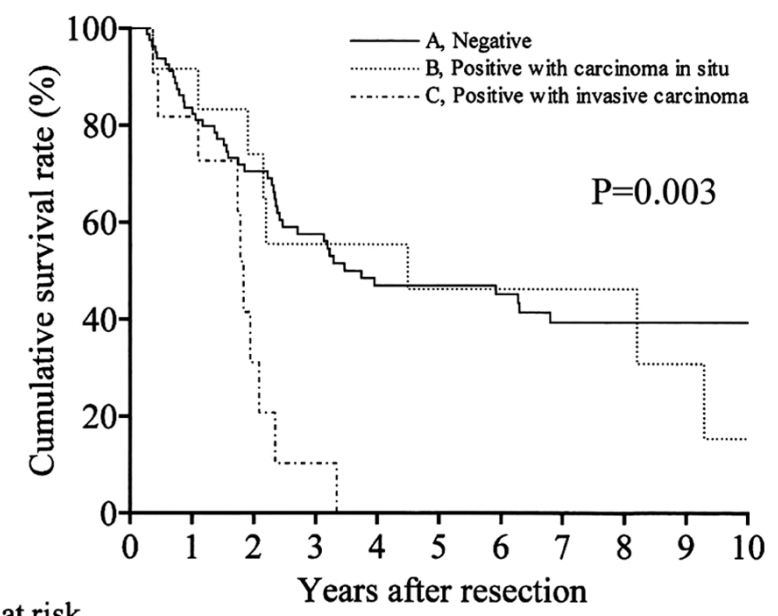

$\begin{array}{lrrrrrrrrrrr}\text { No. at risk } & & & & & & & & & & \\ \text { A } & 85 & 66 & 51 & 39 & 31 & 29 & 26 & 19 & 14 & 12 & 11 \\ \text { B } & 14 & 11 & 8 & 6 & 6 & 5 & 5 & 5 & 4 & 2 & 1 \\ \text { C } & 11 & 9 & 3 & 1 & 0 & 0 & 0 & 0 & 0 & 0 & 0\end{array}$

Figure 1. Kaplan-Meier survival estimates according to ductal margin status. Median survival time was 42,54 and 22 months in patients with negative ductal margins, positive ductal margins with carcinoma in situ, and positive ductal margins with invasive carcinoma, respectively $(\mathrm{P}=0.003)$.

confocal laser scanning microscope (LSM510META; Carl Zeiss, Jena, Germany). The nuclear staining pattern of 53BP1 was classified into 2 categories: diffuse localization implying inactivation of 53BP1, or discrete foci implying activation of 53BP1 (26-28).

Prognostic factors. To elucidate factors influencing long-term outcome after resection, 16 conventional variables were tested in all 110 patients: age ( $<65$ years vs. $\geq 65$ years), gender, preoperative serum carcinoembryonic antigen (CEA) level $(<5 \mathrm{ng} / \mathrm{ml} \mathrm{vs} . \geq 5 \mathrm{ng} / \mathrm{ml}$ ), type of resection (hepatectomy with bile duct resection vs. pancreaticoduodenectomy vs. bile duct resection vs. combined hepatectomy and pancreaticoduodenectomy), adjuvant treatment (absent vs. present), location of the primary tumor (hilar vs. nonhilar), size of the primary tumor ( $<40 \mathrm{~mm}$ vs. $\geq 40 \mathrm{~mm}$ ), pathologic primary tumor classification (pT1 plus pT2 vs. pT3 plus pT4), pathologic lymph node metastasis classification (pN0 vs. pN1), pathologic distant metastasis classification (pM0 vs. pM1), histopathologic type (papillary vs. non-papillary), histologic grade (G1 vs. G2 vs. G3), lymphatic vessel invasion (absent vs. present), blood vessel invasion (absent vs. present), perineural invasion (absent vs. present), and ductal resection margin status (negative vs. carcinoma in situ vs. invasive carcinoma). The cut-off levels for patient age (65 years) and size of the primary tumor $(40 \mathrm{~mm})$ were determined based on the respective median values, whereas the cut-off level of preoperative serum CEA was set at $5 \mathrm{ng} / \mathrm{ml}$, which was the upper limit of the reference range for serum CEA $(<5 \mathrm{ng} / \mathrm{ml})$.

Statistical analysis. Medical records and survival data were obtained for all 110 patients. Categorical variables were compared using Fisher's exact test or the Pearson $\chi^{2}$ test; continuous variables were compared using the Mann-Whitney $U$ test. The
A

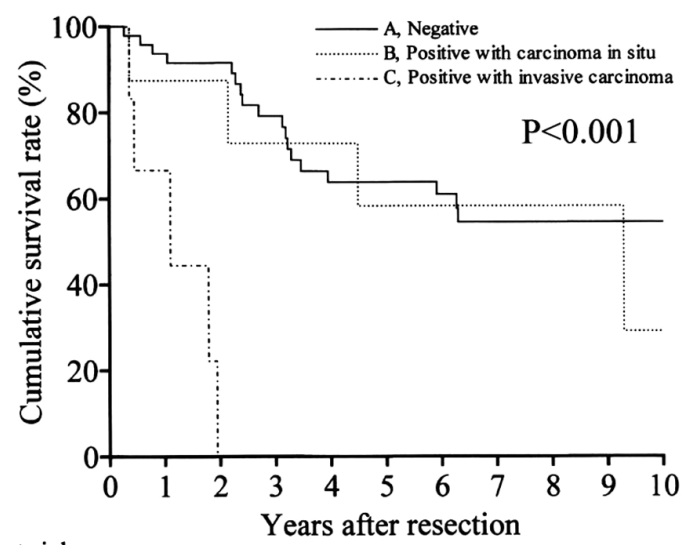

No. at risk

$\begin{array}{llllllllllll}\text { A } & 48 & 45 & 39 & 32 & 25 & 23 & 21 & 15 & 12 & 10 & 9\end{array}$

B

C

$\mathbf{B}$

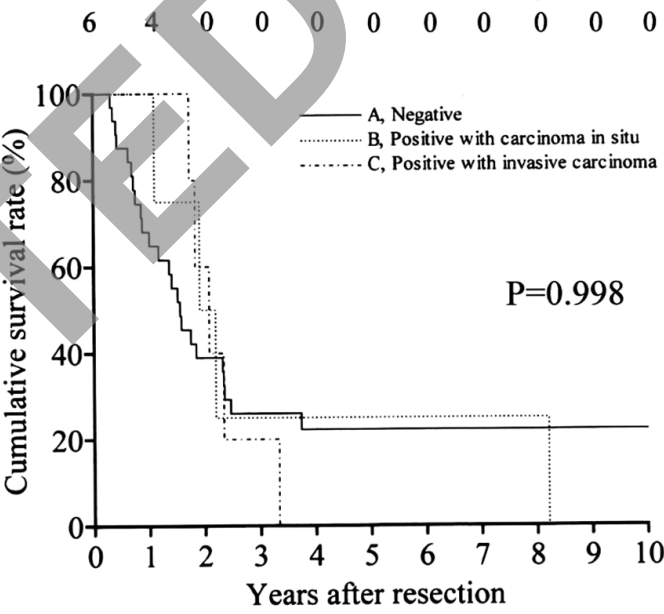

No. at risk

$\begin{array}{lrrrrrrrrrrr}\mathrm{A} & 37 & 21 & 12 & 7 & 6 & 5 & 4 & 3 & 2 & 2 & 2 \\ \mathrm{~B} & 4 & 4 & 2 & 1 & 1 & 1 & 1 & 1 & 1 & 0 & 0 \\ \mathrm{C} & 5 & 5 & 3 & 1 & 0 & 0 & 0 & 0 & 0 & 0 & 0\end{array}$

Figure 2. (A) Kaplan-Meier survival estimates according to ductal margin status in 64 patients that were pathologically negative for nodal and distant metastases (pN0pM0). (B) Kaplan-Meier survival estimates according to ductal margin status in 46 patients that were pathologically positive for nodal and/or distant metastases (pN1 and/or pM1).

causes of death were determined from the medical records. Deaths from cancer and/or suppurative cholangitis were treated as failure cases and those from other causes as censored cases. The Kaplan-Meier method was used to estimate the cumulative incidences of events, and differences in these incidences were evaluated by the log-rank test. The Cox proportional hazards regression model was used to identify factors that were independently associated with survival and local recurrence after resection. In this model, a stepwise selection is used for variable selection, with entry and removal limits of $\mathrm{P}<0.05$ and $\mathrm{P}>0.1$, respectively. All statistical evaluations were performed using the PASW Statistics 17 software package (SPSS Japan Inc., Tokyo, Japan). All tests were two-tailed and a $\mathrm{P}<0.05$ was considered statistically significant.

\section{Results}

Clinicopathologic characteristics according to ductal margin status. The ductal resection margin was negative in 85 patients, 


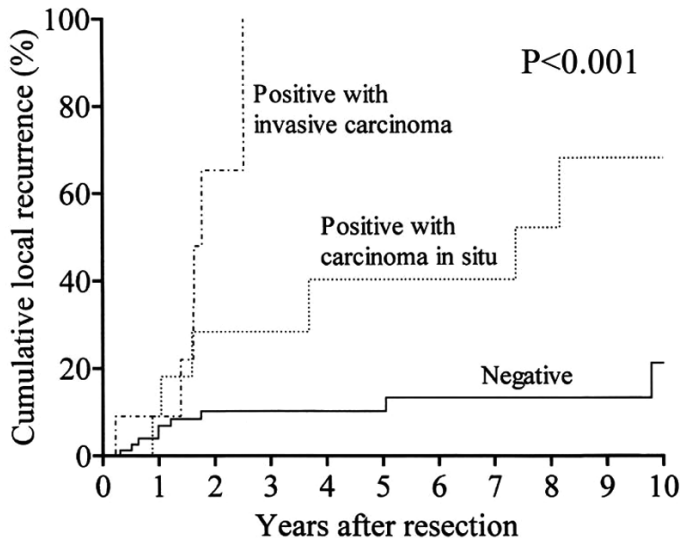

$\begin{array}{lrrrrrrrrrrr}\text { No. at risk } & & & & & & & & & \\ \text { Invasive } & 11 & 9 & 1 & 0 & 0 & 0 & 0 & 0 & 0 & 0 & 0 \\ \text { Carcinoma in situ } & 14 & 10 & 6 & 6 & 5 & 5 & 5 & 5 & 3 & 1 & 1 \\ \text { Negative } & 85 & 64 & 48 & 38 & 31 & 29 & 26 & 19 & 14 & 12 & 10\end{array}$

Figure 3. Kaplan-Meier estimates of local recurrence at ductal stumps. The cumulative probability of local recurrence at 5 years was $10 \%, 40 \%$ and $100 \%$ in patients with negative ductal margins, positive with carcinoma in situ, and positive with invasive carcinoma, respectively $(\mathrm{P}<0.001)$.

positive with carcinoma in situ in 14 patients, and positive with invasive carcinoma in 11 patients. Clinicopathologic characteristics were comparable among the 3 groups with the exception that the type of surgical resection, adjuvant treatment, and perineural invasion were significantly associated with ductal resection margin status. A positive ductal resection margin was more frequent in patients who underwent a resection of the extrahepatic bile duct alone $(\mathrm{P}=0.036)$. Adjuvant treatment was more frequently performed in patients with a positive margin with invasive carcinoma $(\mathrm{P}=0.017)$. Perineural invasion was also more frequent in patients with a positive margin with invasive carcinoma $(\mathrm{P}=0.013)$.

Factors influencing long-term survival after resection. Overall cumulative survival rates after resection were $42 \%$ at 5 years and $31 \%$ at 10 years (median survival time 39 months). Univariate analysis revealed that the size of the primary tumor, $\mathrm{pN}$ classification, pM classification, histologic grade, blood vessel invasion, and ductal margin status were significantly associated with long-term survival (Table I). These six variables were entered into a multivariate analysis, and $\mathrm{pN}$ classification $(\mathrm{P}=0.037), \mathrm{pM}$ classification $(\mathrm{P}=0.001)$, and ductal margin status $(\mathrm{P}=0.029)$ remained independently associated with long-term survival (Table I).

Ductal margin status significantly affected survival after resection ( $\mathrm{P}=0.003$; Fig. 1). When all 110 patients were divided according to their $\mathrm{pN}$ and $\mathrm{pM}$ classifications, further significant differences were observed. Of 64 patients that were pathologically negative for nodal and distant metastases (pNOpM0), ductal margin status appeared to have a strong impact on long-term survival ( $\mathrm{P}<0.001$; Fig. 2A). Of 46 patients that were pathologically positive for nodal and/or distant metastases (pN1 and/ or pM1), the differences in long-term survival between the 3 groups of patients, according to their ductal margin status, were not significant ( $\mathrm{P}=0.998$; Fig. 2B).

Factors influencing local recurrence at ductal stumps. At the time of disease status assessment, tumor recurrences had occurred in 66 patients. The sites of initial disease recurrence were local at ductal stumps $(n=8)$, local at ductal stumps plus distant lymph nodes $(n=2)$, local at ductal stumps plus distant organs $(n=11)$, distant lymph nodes $(n=8)$, and distant organs $(n=37)$. In total, 21 of $110(19 \%)$ patients had clinically evident local recurrences at ductal stumps, which were verified by biopsy and/or bile cytology after percutaneous transhepatic biliary drainage $(n=18)$, computed tomography images $(n=2)$, or a biopsy taken on explorative laparotomy $(n=1)$.

The cumulative probability of local recurrence at 5 years was $10 \%, 40 \%$, and $100 \%$ in patients with negative ductal margins, positive with carcinoma in situ, and positive with invasive carcinoma, respectively $(\mathrm{P}<0.001$; Fig. 3$)$. The median length of time until local recurrence at ductal stumps occurred was 88 months for patients with residual carcinoma in situ, and 21 months for patients with residual invasive carcinoma. Univariate analysis revealed that ductal margin status and

Table II. Factors significantly associated with local recurrence at ductal stumps.

\begin{tabular}{|c|c|c|c|c|c|c|}
\hline \multirow[b]{2}{*}{ Variable } & \multirow[b]{2}{*}{ No. of patients } & \multicolumn{2}{|c|}{$\begin{array}{c}\text { Local recurrence } \\
\text { rate }(\%)\end{array}$} & \multirow{2}{*}{$\begin{array}{l}\text { Univariate } \\
\text { analysis } \\
\text { P-value }\end{array}$} & \multicolumn{2}{|l|}{$\begin{array}{l}\text { Multivariate } \\
\text { analysis }\end{array}$} \\
\hline & & 5-year & 10-year & & Relative risk (95\% CI) & P-value \\
\hline Lymphatic vessel invasion & & & & 0.031 & & \\
\hline Absent & 35 & 8 & 31 & & & \\
\hline Present & 75 & 29 & 44 & & & \\
\hline Ductal margin status & & & & $<0.001$ & & 0.001 \\
\hline Negative & 85 & 10 & 21 & & 1.00 & \\
\hline Positive with carcinoma in situ & 14 & 40 & 68 & & $4.26(1.50-12.08)$ & 0.006 \\
\hline Positive with invasive carcinoma & 11 & 100 & 100 & & $7.00(2.29-21.35)$ & 0.001 \\
\hline
\end{tabular}

CI, confidence interval. 

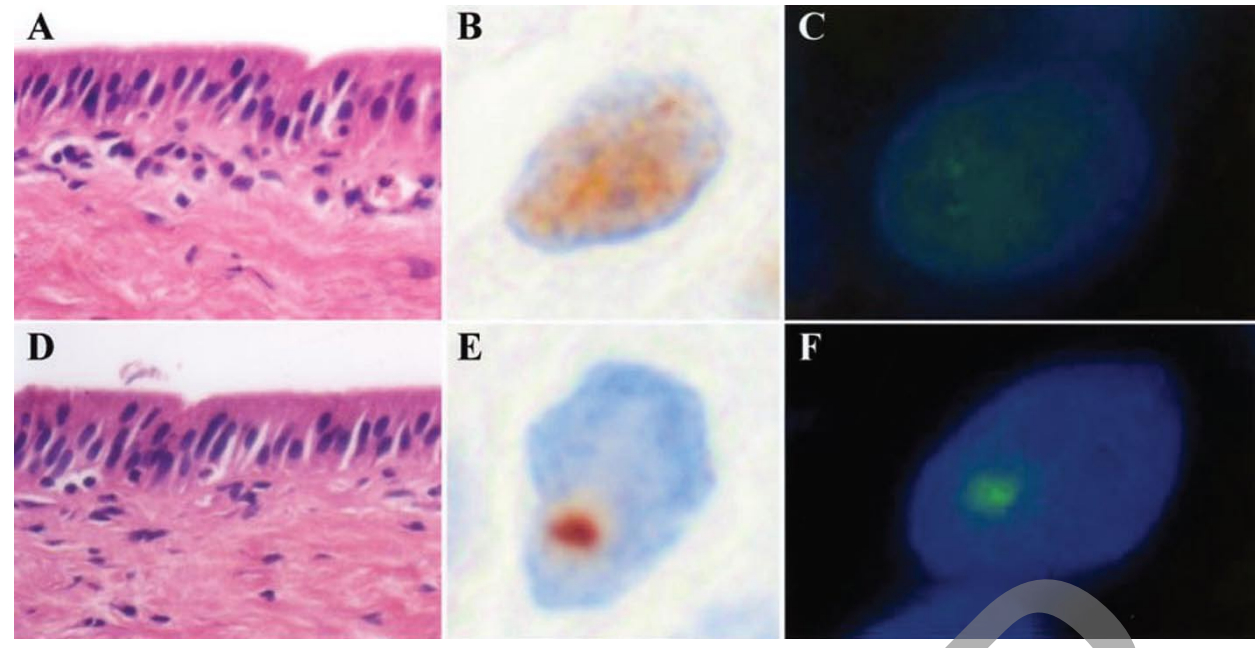

Figure 4. Expression of p53-binding protein 1 (53BP1) in non-neoplastic biliary epithelium. (A) Hematoxylin-eosin staining. (B) Immunohistochemical staining for 53BP1 shows faint-diffuse nuclear staining. (C) Faint-diffuse localization of 53BP1 in nucleus is visualized by immunofluorescence. (D) Hematoxylin-eosin staining. (E) Immunohistochemical staining for 53BP1 shows one discrete nuclear focus. (F) One discrete nuclear focus of 53BP1 is visualized by immunofluorescence. Immunofluorescence images ( $\mathrm{C}$ and F), green 53BP1; blue DAPI.

lymphatic vessel invasion were significantly associated with local recurrence after resection (Table II). These two variables were entered into a multivariate analysis, and ductal resection margin status was the only independent risk factor for local recurrence $(\mathrm{P}=0.001$; Table II).

Expression of 53BP1 in non-neoplastic biliary epithelium. Of the 7 samples of non-neoplastic biliary epithelium used as normal controls (Fig. 4A-F), 6 showed faint-diffuse nuclear staining of 53BP1 (Fig. 4B and C), whereas 1 showed one or two discrete nuclear foci of 53BP1 (Fig, 4E and F).

DNA damage response in tumor specimens. The DNA damage response in tumor specimens of ductal resection margins (14 tumor specimens of carcinoma in situ vs. 11 tumor specimens of invasive carcinoma) was evaluated by immunohistochemistry, TUNEL, and immunofluorescence. Labeling indices for p53 ( $\mathrm{P}=0.732$; Fig. 5A) and Ki-67 (P=0.168; Fig. 5B) were not apparently different between carcinoma in situ and invasive carcinoma. The level of DNA DSBs, based on the $\gamma \mathrm{H} 2 \mathrm{AX}$ labeling index, was significantly higher in invasive carcinoma than carcinoma in situ tumor specimens $(\mathrm{P}=0.031$; Fig. 5C), while the apoptotic index was significantly lower in invasive carcinoma than carcinoma in situ specimens $(\mathrm{P}=0.004$; Fig. 5D). The incidence of $\mathrm{p} 53$ overexpression was comparable between tumor specimens of carcinoma in situ [8 of $14(57 \%)$ ] and tumor specimens of invasive carcinoma [6 of $11(55 \%)$; $\mathrm{P}>0.999]$.

All the 11 tumor specimens of invasive carcinoma showed diffuse localization of 53BP1 in nuclei. Of the 14 tumor specimens of carcinoma in situ (Fig. 6A-H), 10 were classified as having tumors with diffuse localization of 53BP1 in nuclei (Fig. 6B and C) and 4 had discrete nuclear foci of 53BP1 (Fig. 6F and $\mathrm{G})$. The nuclear staining pattern of 53BP1 was more clearly visualized by immunofluorescence analysis (Fig. 6C and G) than immunohistochemistry (Fig. 6B and F). In the 21 tumor specimens with diffuse localization of 53BP1 in nuclei, co-
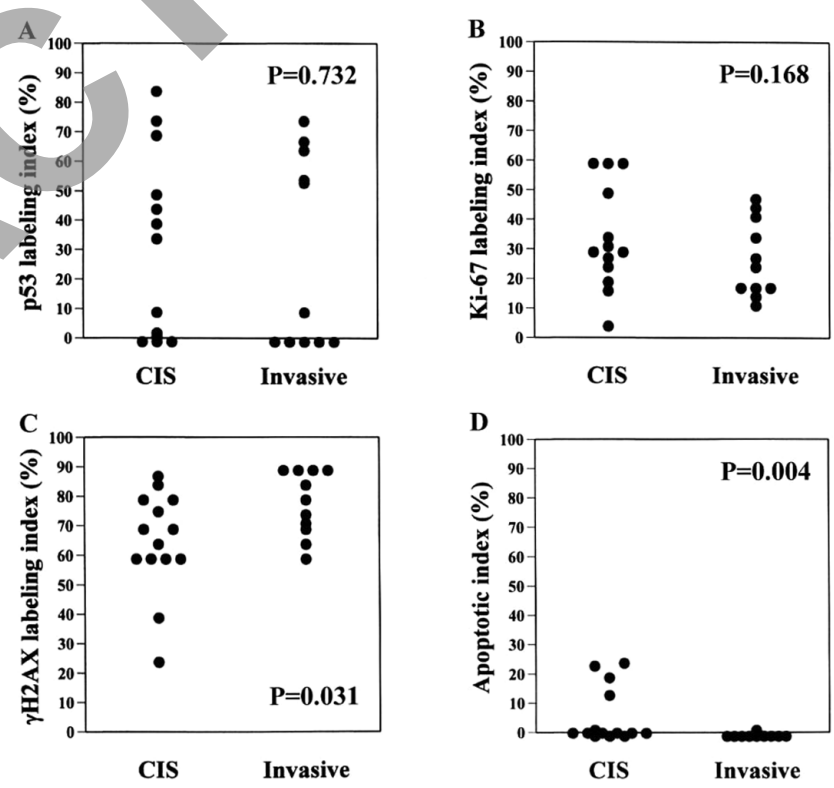

Figure 5. Labeling index of DNA damage response-protein in tumor specimens of ductal resection margins. (A) p53 labeling index. (B) Ki-67 labeling index. (C) $\gamma \mathrm{H} 2 \mathrm{AX}$ labeling index. (D) Apoptotic index. CIS, carcinoma in situ; invasive, invasive carcinoma.

localization of 53BP1 with $\gamma \mathrm{H} 2 \mathrm{AX}$ at sites of DSBs was not observed by double-labeled immunofluorescence, indicating 53BP1 inactivation. In the 4 tumor specimens with discrete nuclear foci of 53BP1, co-localization of 53BP1 with $\gamma \mathrm{H} 2 \mathrm{AX}$ at sites of DSBs was confirmed by double-labeled immunofluorescence (Fig. 7), indicating 53BP1 activation.

The median values of apoptotic index were $0 \%, 1 \%$ and $22 \%$ in tumor specimens of invasive carcinoma with 53BP1 inactivation, carcinoma in situ with 53BP1 inactivation, and carcinoma in situ with 53BP1 activation, respectively. The apoptotic index was significantly lower in the 21 tumor specimens with 53BP1 inactivation (11 specimens of invasive 

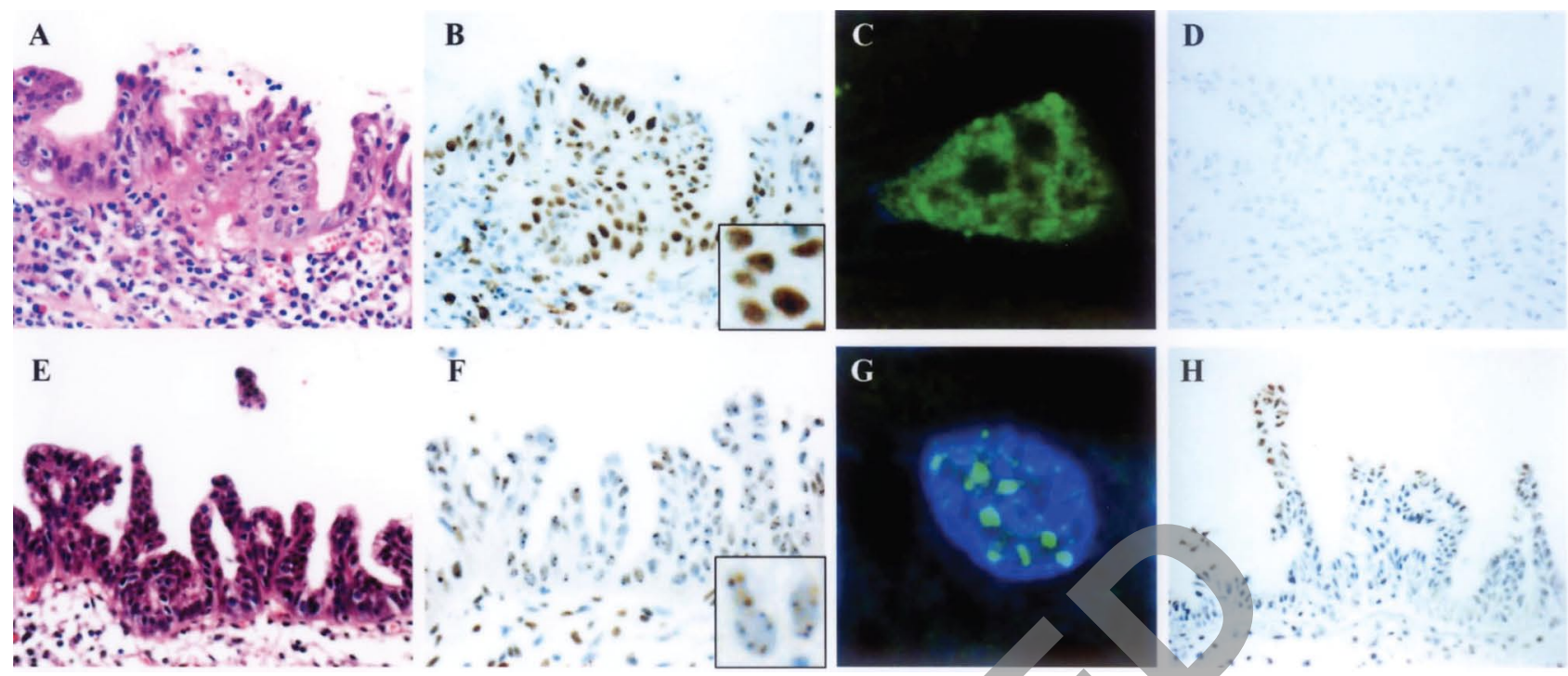

Figure 6. Nuclear staining pattern of p53-binding protein 1 (53BP1) in tumor specimens of carcinoma in situ. (A) Hematoxylin-eosin staining. (B) Immunohistochemical staining for 53BP1 shows diffuse localization of 53BP1 in the nuclei. (C) Diffuse localization of 53BP1 in nucleus is visualized by immunofluorescence. (D) No apoptotic cells are observed by TUNEL analysis. (E) Hematoxylin-eosin staining. (F) Immunohistochemical staining for 53BP1 shows discrete nuclear foci of 53BP1. (G) Discrete nuclear foci of 53BP1 are visualized by immunofluorescence. (H) Apoptotic cells are observed by TUNEL analysis. Immunofluorescence images (C and G), green 53BP1; blue DAPI.

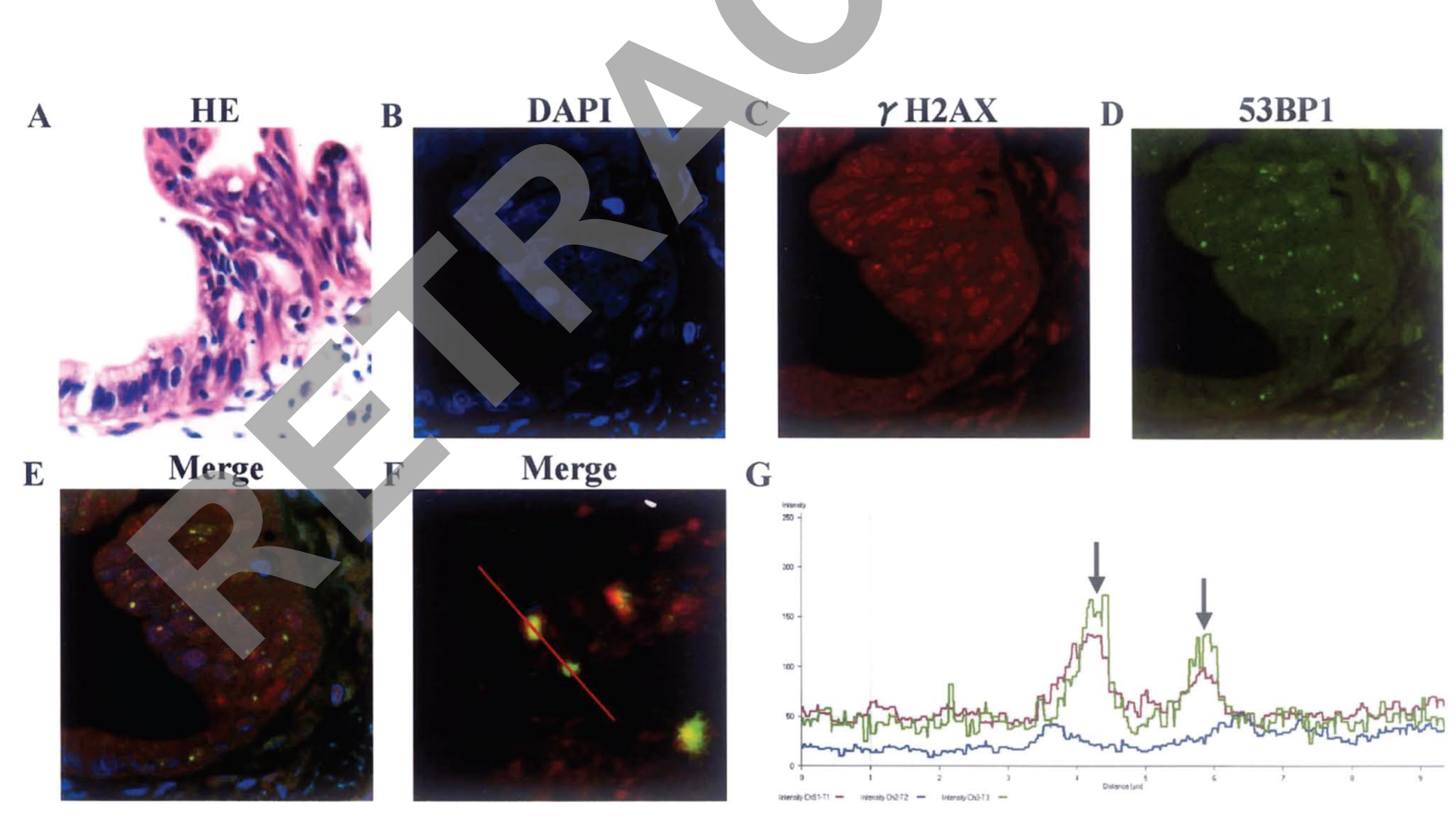

Figure 7. Double-labeled immunofluorescence with $\gamma \mathrm{H} 2 \mathrm{AX}$ and p53-binding protein 1 (53BP1) antibodies in a tumor specimen of carcinoma in situ with discrete nuclear foci of 53BP1. (A) Hematoxylin-eosin staining (HE). (B) Blue DAPI. (C) Red $\gamma \mathrm{H} 2 \mathrm{AX}$. (D) Green 53BP1. (E) A merged image depicts 53BP1 foci predominantly co-localize with $\gamma \mathrm{H} 2 \mathrm{AX}$ foci in the nuclei. (F) High magnification of (E). (G) The intensity curves are visualized through the length of the red line in (F). The 2 peaks (arrows) of the green intensity curve of 53BP1 co-localize with the 2 peaks of the red intensity curve of $\gamma \mathrm{H} 2 \mathrm{AX}$.

carcinoma and 10 specimens of carcinoma in situ) compared to the 4 tumor specimens of carcinoma in situ with 53BP1 activation (median index, $0 \%$ vs. $22 \%$; $\mathrm{P}<0.001$ ). The apoptotic index was significantly lower in the 10 tumor specimens of carcinoma in situ with 53BP1 inactivation (Fig. 6D) than in the 4 tumor specimens of carcinoma in situ with 53BP1 activation (Fig. 6H) (P=0.001; Fig. 8). The incidence of p53 overexpression was comparable between tumor specimens with 53BP1 inactivation [12 of $21(57 \%)$ ] and 53BP1 activation [2 of 4 (50\%); P>0.999]. All the main tumors from the 25 patients with positive ductal margins showed diffuse localization of 53BP1 in the nuclei (53BP1 inactivation). 


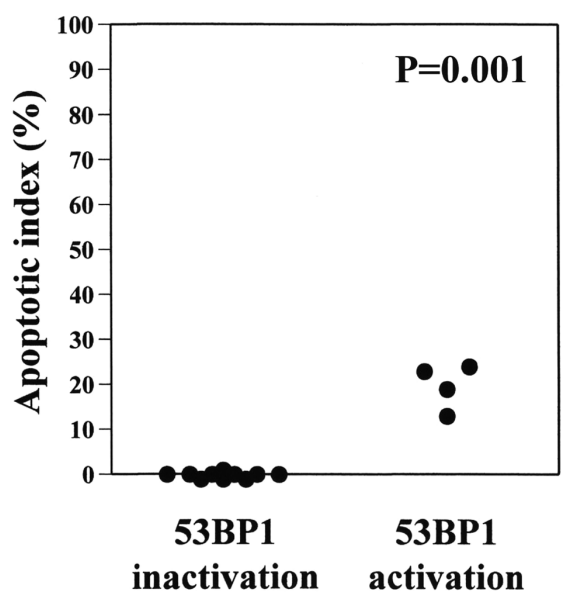

Figure 8. Apoptotic index in tumor specimens of carcinoma in situ according to 553 -binding protein 1 (53BP1) status.

Impact of 53BP1 status on long-term outcome after resection in patients with residual carcinoma in situ. Local recurrences at ductal stumps were observed in 6 of 10 patients with residual carcinoma in situ with 53BP1 inactivation. Among the 14 patients with positive ductal margins with carcinoma in situ, the cumulative probability of local recurrence was significantly higher in patients with 53BP1 inactivation than in patients with 53BP1 activation (cumulative 5-year local recurrence rate, $60 \%$ vs. $0 \%$, respectively; $\mathrm{P}=0.020$; Fig. $9 \mathrm{~A}$ ). Cumulative survival after resection in these 14 patients was significantly worse in those with 53BP1 inactivation than in those with 53BP1 activation (cumulative 10-year survival rate, $0 \%$ vs. $100 \%$, respectively; $\mathrm{P}=0.026$; Fig. 9B).

\section{Discussion}

Although ductal margin status is an established prognostic factor in patients with extrahepatic cholangiocarcinoma (17), a small number of patients with positive ductal margins with carcinoma in situ still survive in the long-term (7-16). This fact inspired the current study which focused on evaluation of the early 53BP1-mediated DNA damage response by immunofluorescence analysis in tumor specimens of ductal resection margins and local recurrence at ductal stumps. To our knowledge, the current study is the first to demonstrate that clinically evident local recurrence at ductal stumps is closely associated with 53BP1 inactivation (diffuse localization of 53BP1 in nuclei) and decreased apoptosis in tumor specimens of ductal resection margins. In contrast, 53BP1 activation (discrete nuclear foci of 53BP1) in residual carcinoma in situ at ductal stumps may result in long-term postresection survival with no evidence of local recurrence. Thus, immunofluorescence analysis of 53BP1 nuclear expression in tumor specimens of ductal resection margins may be useful to estimate the risk for local recurrence at ductal stumps.

Recent studies have demonstrated increased levels of DNA DSBs not only in tumors but also in precancerous lesions in clinical specimens from various tissues (2,26,29-31). In the current study, invasive carcinoma at ductal resection margins showed a markedly high $\gamma \mathrm{H} 2 \mathrm{AX}$-labeling index and 53BP1
A

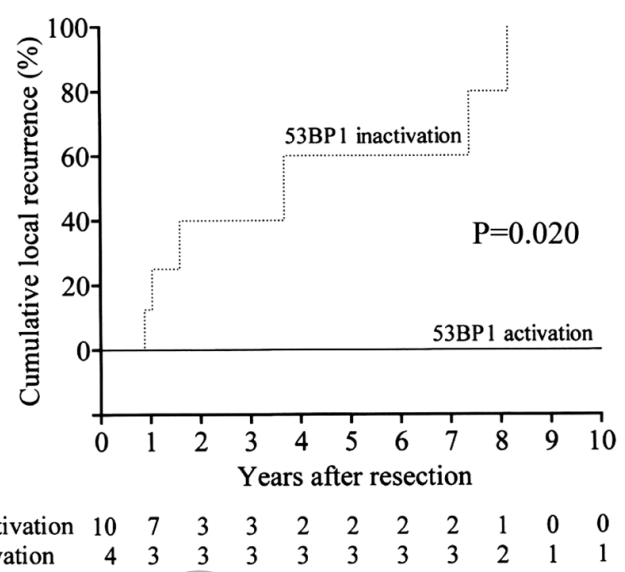

$\begin{array}{lrrrrrrrrrrr}\text { 53BP1 inactivation } & 10 & 7 & 3 & 3 & 2 & 2 & 2 & 2 & 1 & 0 & 0 \\ \text { 53BP1 activation } & 4 & 3 & 3 & 3 & 3 & 3 & 3 & 3 & 2 & 1 & 1\end{array}$

B

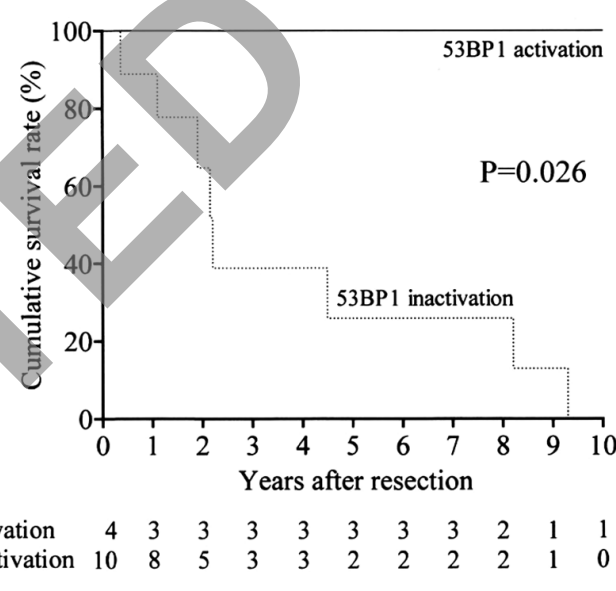

Figure 9. (A) Kaplan-Meier estimates of local recurrence at ductal stumps in patients with residual carcinoma in situ according to p53-binding protein 1 (53BP1) status. (B) Kaplan-Meier survival estimates in patients with residual carcinoma in situ according to 53BP1 status.

inactivation, suggesting an inefficient 53BP1-mediated DNA damage response. Carcinoma in situ at ductal resection margins could clearly be subdivided into 2 categories according to the nuclear staining pattern of 53BP1 by immunofluorescence. Carcinoma in situ with diffuse localization of 53BP1 in nuclei showed decreased apoptosis and was associated with a high incidence of local recurrence at ductal stumps, suggesting that clinically evident local recurrence at ductal stumps (i.e., the progression of residual carcinoma in situ to invasive carcinoma at ductal resection margins) is associated with 53BP1 inactivation and decreased apoptosis. In contrast, carcinoma in situ with discrete nuclear foci of 53BP1 showed increased apoptosis and was associated with a favorable survival with no evidence of local recurrence, suggesting that 53BP1 activation and increased apoptosis in residual carcinoma in situ at ductal stumps may result in long-term postresection survival. Taken together, our findings suggest that an inefficient 53BP1mediated DNA damage response has a crucial role in the progression of residual carcinoma in situ to invasive carcinoma at ductal stumps.

The incidence of local recurrence at ductal stumps was $19 \%$ in the current series, and was associated with positive ductal margins. Some authors $(8,11,13)$ reported a significant relationship between local recurrence and ductal margin status based on statistical analysis of the frequency of local 
recurrence according to ductal margin status. As tumor recurrence depends on follow-up time, we applied the Cox proportional hazards regression model to identify risk factors for local recurrence at ductal stumps, and ductal resection margin status was found to be the only independent risk factor for local recurrence. Earlier reports suggested that residual carcinoma in situ does not have a strong adverse effect on patient survival and may cause late local recurrence (7-16). New results from our current study found that the relative risk for local recurrence in patients with residual carcinoma in situ was 4.26 , and 10 of $14(71 \%)$ tumor specimens of carcinoma in situ had an inefficient 53BP1-mediated DNA damage response and the likelihood of local recurrence. Therefore, we believe that residual carcinoma in situ with inefficient 53BP1mediated DNA damage response has the potential to progress to invasive carcinoma.

Recent surgical studies reported that limited additional resection $(\leq 5 \mathrm{~mm})$ of a margin-positive proximal bile duct did not lead to improvement in survival of patients with hilar cholangiocarcinoma, even if negative margins were achieved with the additional resection $(12,32)$. Possible reasons for the poor prognosis in patients who achieved negative proximal margins after additional resection have been proposed $(10,12)$. Firstly, all such cases have 1 to 4 independent adverse prognostic factors $(10,12)$, and secondly, in such cases, clearance of the proximal margin may offer no survival benefit because the ductal margin status is a less powerful prognostic factor in hilar cholangiocarcinoma (12). Our current results found that after stratification according to $\mathrm{pN}$ and $\mathrm{pM}$ classification, ductal resection margin status significantly affected long-term survival after resection for patients with pNOpM0 disease, but not for patients with $\mathrm{pN} 1$ and/or pM1 disease. Taken together, these findings suggest that in patients with pN1 and/or pM1 disease, ductal resection margin status appears to have no impact on long-term survival. When ductal margin status is found to be positive on frozen-section examination, additional resection of the bile duct should be considered in early-stage disease (pN0pM0 disease) or minimally invasive papillary cancer (10,33-35). Further investigation is required to elucidate the effect of additional resection of margin-positive bile ducts on the survival of patients with non-hilar cholangiocarcinoma.

The current study has some limitations. Firstly, it was a retrospective analysis of a small number of patients with positive ductal margins $(n=25)$, and secondly, the follow-up time was shorter than 5 years in 29 patients. However, we believe that these limitations did not significantly influence the outcome of the study due to the fact that the differences among groups were too marked to have resulted from these biases. The current study has a potential clinical implication. Immunofluorescence analysis of 53BP1 expression can be performed on tumor specimens to evaluate the early DNA damage response (inefficient response or efficient response), which may be a useful histologic marker to estimate the potential for clinically malignant behavior in precancerous lesions of the biliary tract, as well as in a variety of other human tumors $(3,5,6,29-31,36)$.

In conclusion, after resection for extrahepatic cholangiocarcinoma, clinically evident local recurrence at ductal stumps is closely associated with 53BP1 inactivation and decreased apoptosis in tumor specimens of ductal resection margins.
Immunofluorescence analysis of 53BP1 nuclear expression in tumor specimens of ductal resection margins may be useful to estimate the risk for local recurrence at ductal stumps in patients undergoing resection for extrahepatic cholangiocarcinoma.

\section{Acknowledgements}

This study was supported in part by a Grant-in-Aid for Scientific Research no. 21591768 (T.W. and Y.S.) from the Ministry of Education, Culture, Sports, Science, and Technology of Japan.

\section{References}

1. Bonner WM, Redon CE, Dickey JS, et al: $\gamma \mathrm{H} 2 \mathrm{AX}$ and cancer. Nat Rev Cancer 8: 957-967, 2008.

2. Difilippantonio S, Gapud E, Wong N, et al: 53BP1 facilitates long-range DNA end-joining during V(D)J recombination. Nature 456: 529-533, 2008.

3. Gorgoulis VG, Vassiliou LV, Karakaidos P, et al: Activation of the DNA damage checkpoint and genomic instability in human precancerous lesions. Nature 434: 907-913, 2005.

4. Stavnežer J, Guikema JE and Schrader CE: Mechanism and regulation of class switch recombination. Annu Rev Immunol 26: 261-292, 2008

5. Nakashima M, Suzuki K, Meirmanov S, et al: Foci formation of P53-binding protein 1 in thyroid tumors: activation of genomic instability during thyroid carcinogenesis. Int J Cancer 122: $1082-1088,2008$.

6. Naruke Y, Nakashima M, Suzuki K, et al: Alteration of p53-binding protein 1 expression during skin carcinogenesis: association with genomic instability. Cancer Sci 99: 946-951, 2008.

7. Wakai T, Shirai Y, Moroda T, Yokoyama N and Hatakeyama K: Impact of ductal resection margin status on long-term survival in patients undergoing resection for extrahepatic cholangiocarcinoma. Cancer 103: 1210-1216, 2005.

8. Sasaki R, Takeda Y, Funato O, et al: Significance of ductal margin status in patients undergoing surgical resection for extrahepatic cholangiocarcinoma. World J Surg 1: 1788-1796, 2007.

9. Ojima H, Kanai Y, Iwasaki M, et al: Intraductal carcinoma component as a favorable prognostic factor in biliary tract carcinoma. Cancer Sci 100: 62-70, 2009.

10. Igami T, Nagino M, Oda K, et al: Clinicopathologic study of cholangiocarcinoma with superficial spread. Ann Surg 249: 296-302, 2009.

11. Nakanishi Y, Kondo S, Zen Y, et al: Impact of residual in situ carcinoma on postoperative survival in 125 patients with extrahepatic bile duct carcinoma. J Hepatobiliary Pancreat Sci 17: 166-173, 2010.

12. Shingu Y, Ebata T, Nishio H, Igami T, Shimoyama Y and Nagino M: Clinical value of additional resection of a marginpositive proximal bile duct in hilar cholangiocarcinoma. Surgery 147: 49-56, 2010.

13. Higuchi R, Ota T, Araida T, Kobayashi M, Furukawa T and Yamamoto M: Prognostic relevance of ductal margins in operative resection of bile duct cancer. Surgery 148: 7-14, 2010.

14. Nakanishi Y, Kondo S, Hirano S, et al: Recurrence of mucosal carcinoma of the bile duct, with superficial flat spread, 12 years after operation. J Hepatobiliary Pancreat Surg 13: 355-358, 2006.

15. Sasaki T, Kondo S, Ambo Y, et al: Local recurrence at hepaticojejunostomy 9 years after resection of bile duct cancer with superficial flat spread. J Hepatobiliary Pancreat Surg 13: 458-462, 2006.

16. Nakanishi Y, Zen Y, Kawakami H, et al: Extrahepatic bile duct carcinoma with extensive intraepithelial spread: a clinicopathological study of 21 cases. Mod Pathol 21: 807-816, 2008.

17. Greene FL, Page DL, Fleming ID, et al: American Joint Committee on Cancer Staging Manual. 6th edition, SpringerVerlag, New York, pp145-150, 2002.

18. Shirai Y, Yoshida K, Tsukada K and Muto T: Inapparent carcinoma of the gallbladder. An appraisal of a radical second operation after simple cholecystectomy. Ann Surg 215: 326-331, 1992.

19. Shirai Y, Yoshida K, Tsukada K, Ohtani T and Muto T: Identification of the regional lymphatic system of the gallbladder by vital staining. Br J Surg 79: 659-662, 1992. 
20. Shirai Y, Ohtani T, Tsukada K and Hatakeyama K: Patterns of lymphatic spread of carcinoma of the ampulla of Vater. Br J Surg 84: 1012-1016, 1997

21. Bismuth H, Nakache R and Diamond T: Management strategies in resection for hilar cholangiocarcinoma. Ann Surg 215: 31-38, 1992.

22. Bismuth $\mathrm{H}$ and Corlette MB: Intrahepatic cholangioenteric anastomosis in carcinoma of the hilus of the liver. Surg Gynecol Obstet 140: 170-178, 1975.

23. Albores-Saavedra J, Henson DE and Klimstra DS: Tumors of the gallbladder, extrahepatic bile ducts, and ampulla of Vater. 3rd edition, Armed Forces Institute of Pathology, Washington, DC pp191-215, 2000

24. Albores-Saavedra J, Henson DE and Sobin LH: Histological typing of tumours of the gallbladder and extrahepatic bile ducts. In: World Health Organization. International Histological Classification of Tumours. Springer-Verlag, Berlin, 1991.

25. Oohashi Y, Watanabe H, Ajioka Y and Hatakeyama K: p53 immunostaining distinguishes malignant from benign lesions of the gall-bladder. Pathol Int 45: 58-65, 1995.

26. Nuciforo PG, Luise C, Capra M, Pelosi G and d'Adda di Fagagna F: Complex engagement of DNA damage response pathways in human cancer and in lung tumor progression. Carcinogenesis 28: 2082-2088, 2007.

27. Rappold I, Iwabuchi K, Date T and Chen J: Tumor suppressor p53 binding protein 1 (53BP1) is involved in DNA damagesignaling pathways. J Cell Biol 153: 613-620, 2001.

28. Schultz LB, Chehab NH, Malikzay A and Halazonetis TD: p53 binding protein 1 (53BP1) is an early participant in the cellular response to DNA double-strand breaks. J Cell Biol 151: 1381-1390, 2000 .
29. Bartkova J, Horejsí Z, Koed K, et al: DNA damage response as a candidate anti-cancer barrier in early human tumorigenesis. Nature 434: 864-870, 2005

30. Sedelnikova OA and Bonner WM: GammaH2AX in cancer cells: a potential biomarker for cancer diagnostics, prediction and recurrence. Cell Cycle 5: 2909-2913, 2006.

31. Yu T, MacPhail SH, Banáth JP, Klokov D and Olive PL: Endogenous expression of phosphorylated histone $\mathrm{H} 2 \mathrm{AX}$ in tumors in relation to DNA double-strand breaks and genomic instability. DNA Repair 5: 935-946, 2006.

32. Endo I, House MG, Klimstra DS, et al: Clinical significance of intraoperative bile duct margin assessment for hilar cholangiocarcinoma. Ann Surg Oncol 15: 2104-2112, 2008.

33. Albores-Saavedra J, Murakata L, Krueger JE and Henson DE: Noninvasive and minimally invasive papillary carcinomas of the extrahepatic bile ducts. Cancer 89: 508-515, 2000.

34. Jarnagin WR, Bowne W, Klimstra DS, et al: Papillary phenotype confers improved survival after resection of hilar cholangiocarcinoma. Ann Surg 241: 703-712, 2005.

35. Nagahashi M, Shirai Y, Wakai T, et al: Depth of invasion determines the postresectional prognosis for patients with $\mathrm{T} 1$ extrahepatic cholangiocarcinoma. Cancer 116: 400-405, 2010.

36. Risques RA, Lai LA, Brentnall TA, et al: Ulcerative colitis is a disease of accelerated colon aging: evidence from telomere attrition and DNA damage. Gastroenterology 135: 410-418, 2008. 